\title{
What is the role of the corpus callosum in intermanual transfer of motor skills? A study of three cases with callosal pathology
}

\begin{abstract}
Intermanual transfer for a skilled motor task was studied in two patients with total callosal agenesis, and one with an acquired partial callosal lesion and clinical evidence for disturbed transfer of motor signals. Patients had to draw meaningless figures with one upper extremity (original learning, OL) and to reproduce their mirror-reversals thereafter with the other side (transfer learning, TL). Both directions of intermanual transfer were tested in two conditions, that is, between either proximal or distal muscle groups. Transfer was evaluated by comparing OL and TL performance at the same effector. The main variable of interest was movement time during the first eight trials of OL and TL. All three patients displayed a significant benefit for transfer from the dominant to the non-dominant hand but not vice versa during proximal motor activity. When compared with the performance of healthy subjects tested in almost identical conditions in a previously reported study, the proximal transfer behavior was found to be similar for all patients and the normal group. Although patients exhibited no significant benefit for distal transfer, their non-dominant-to-dominant distal transfer was above the normal range. The similar transfer pattern of the patients and healthy subjects when using proximal musculature suggests that proximal transfer may be subserved by identical extracallosal pathways, most probably by the ipsilaterally descending motor systems. Since non-dominantto-dominant distal transfer was found to be disadvanta-
\end{abstract}

G. Thut $($ ) M. Regard · K.L. Leenders

Neurology Department, Neuropsychological Unit,

University Hospital Zurich, Frauenklinikstrasse 26,

$\mathrm{CH}-8091$ Zurich, Switzerland;

Fax: +41-1-255 4429

G. Thut $\cdot$ K.L. Leenders

Paul Scherrer Institute, Villigen, Switzerland

U. Halsband

Department of Neurology, University Hospital,

Düsseldorf, Germany

E. Mayer · T. Landis

Department of Neurology, University Hospital,

Geneva, Switzerland geous in healthy subjects, the patients' relative superiority in this condition may reflect missing callosal influences of an inhibitory nature.

Key words Callosal pathology $\cdot$ Motor transfer . Distal and proximal movements - Interhemispheric communication - Ipsilateral motor pathways

\section{Introduction}

Intermanual transfer of training has been assessed in a variety of tasks with respect to transfer direction (e.g. Parlow and Kinsbourne 1989). We recently investigated intermanual transfer of a motor skill as a function of transfer direction and muscle group involved (Thut et al. 1996). The results revealed a benefit for transfer between proximal but not distal muscle groups (proximal transfer advantage) when movement time was evaluated. Moreover, right-sided distal relearning was observed to be worsened by previous, contralateral training (right distal transfer disadvantage).

It has been shown that intermanual transfer may require intact callosal fibres (e.g. Lassonde et al. 1986; Levin et al. 1993). Anatomical and neurophysiological investigations, furthermore, revealed a proximal-distal gradient in callosal motor area interconnectivity in monkeys (Pandya and Vignolo 1971; Gould et al. 1986) and transcallosal facilitatory and inhibitory effects between opposite pyramidal tract cells in cats (e.g. Nakamura et al. 1971) or cortical motor areas in humans (e.g. Meyer et al. 1995). While these findings provide indirect evidence for callosal involvement in the proximal transfer advantage and the right distal transfer disadvantage, transfer may also be carried out by ipsilaterally descending motor pathways. Ipsilateral control has been inferred to be involved during unilateral proximal but not distal motor activity in split-brain monkeys (Brinkman and Kuypers 1973), patients with callosal pathology (Gazzaniga et al. 1967) and healthy subjects (Colebatch and Gandevia 1989; Harrison 1991; Mack et al. 1993). While 
cerebral control over ipsilateral muscles may normally engage callosal fibres and direct control may become activated only after callosal lesions, it cannot be ruled out that ipsilateral control might be brought about without the aid of the corpus callosum in healthy subjects as well. Ipsilateral pathways from motor areas to proximal effectors thus represent an alternative anatomical substrate for the proximal transfer advantage.

The aim of the present study was to establish the role of the corpus callosum in the expression of the proximal transfer advantage and right distal transfer disadvantage. Transfer performance was assessed in two patients with total callosal agenesis and in one patient with a partial callosal lesion and clinical evidence for disturbed transfer of motor signals.

\section{Materials and methods}

Subjects

\section{Patient $O . G$.}

The medical history of O.G. was uneventful until the age of 23 years, when he had Leptospira meningitis. From the age of 34 years he suffered from focal seizures with occasional generalization. The electroencephalogram (EEG) showed epileptic activity in the right temporal lobe. One year later, magnetic resonance imaging revealed complete callosal agenesis with associated eversion of the gyri cinguli, deformed lateral ventricles and hypoplasia of the vermis and cerebellum bilaterally. The anterior commissure was intact. At age 47 years O.G. fell, probably during a seizure, from a bicycle and suffered mild brain injury.

When O.G. was tested he was 49 years old and fully employed as an unskilled worker. Neurological status was normal, except for epilepsy with infrequent seizures despite anticonvulsive medication. Neuropsychological evaluation revealed dyslexia (writing and reading problems), impaired short-term memory and slightly reduced executive functions. With the exception of impaired bimanual coordination [reciprocal, simultaneous stretching and clenching of the fists (Luria 1980)], no "split-brain" manifestations were found. Tachistoscopic lexical decision tasks showed left hemisphere language dominance in this right-handed patient. The laterality index was 0.9 (Bryden 1977). The IQ was 95.

\section{Patient A.D.}

The medical history of A.D. was uneventful until the age of 53 years. He then had a neurological examination for temporary panic attacks following an operation for bladder carcinoma. On magnetic resonance imaging, total agenesis of the corpus callosum and a left fronto-paramedian extracerebral cyst were found. The anterior commissure was intact. Neurological status including EEG was normal.

A.D. was tested at the age of 54 years. He was then working as a postman. Mental status examination revealed mild impairment of memory and conceptual thinking. Except for motor sequencing difficulties in bimanual conditions, no evidence of callosal transfer problems were found. According to tachistoscopic testing, language functions were not lateralized. A.D. is right-handed with a laterality index of 0.8 (Bryden 1977). The IQ was 100.

\section{Patient M.M.}

At the age of 39 years, M.M. was hospitalized because of a car accident. On admission, he had a Glasgow Coma Scale of 5. A com- puted tomography scan showed a haematoma in the corpus callosum, localized mostly in the median part, sparing the genu, the ventral splenium and the cerebral hemispheres. Six months later an aneurysm of the right pericallosal artery was clipped. On neuropsychological evaluation, the initially mutistic patient displayed memory impairment and a disconnection syndrome which did not affect visual transfer. The early disconnection syndrome was severe and included an ideomotor apraxia, agraphia, tactile anomia and tactile alexia on the left side, as well as diagonistic dyspraxia. Two years later, most of the symptoms had cleared. Twelve years later, bimanual coordination was evaluated in detail, revealing disturbed transfer of motor signals (Mayer et al. 1991). A left-handed agraphia and memory impairment also persisted up to the time of testing.

At time of testing M.M. was 56 years old and was employed as a supervised, unskilled worker. Neurological examination revealed an unsteady walk. Findings were normal for the upper extremity. M.M. showed inconsistent hand preference in a 10 -item questionnaire (laterality indices - 0.1 Bryden 1977). A more comprehensive questionnaire (Steenhuis and Bryden 1989) revealed a clear bias for left-handed throwing and right-handed writing and drawing. Because there are handedness groups showing dissociated hand preference for fine manual (writing, drawing) as compared with strength/whole arm (throwing) activities (Peters and Murphy 1992), M.M. was classified as left-handed for proximal but righthanded for distal motor activities. The IQ was 114.

\section{Experimental conditions}

The task consisted of drawing meaningless figures on a blank sheet of paper within two horizontal lines using either proximal or distal muscle groups. The subjects were instructed to draw the figures as quickly and accurately as possible; speed was emphasized. To prevent movements at the fingers and wrist in the proximal condition, drawings had to be performed over $20 \mathrm{~cm}$ (line separation) with the pen held by the whole fist without placing the lower arm on the desk. To minimize movements of the shoulder and elbow in the distal condition, the subjects were requested to make their drawings $2 \mathrm{~cm}$ high (line separation) and to draw in classical writing technique with the lower arm fixed on the desk by the contralateral hand. Subjects furthermore had full visual feedback over their drawing hand. However, the pen used was inkless and produced no visible trace.

The experiment comprised 16 blocks, each consisting of the drawing of an unknown figure with one hand (original learning, OL) and immediate reproduction of the vertical mirror-reversal with the contralateral hand (transfer learning, TL). Mirror-image tasks were originally chosen to preserve muscle involvement and the sequence of muscle activation. To reduce additional cognitive processing (e.g. mental rotation, memory), target figures were clearly visible during reproduction. Before OL, six tracing trials were completed with the same hand to introduce the subjects to the new figure. OL consisted of 30 trials. TL performance was recorded for the first eight trials only, since previous research with normal subjects has revealed transfer effects to occur primarily at the beginning of TL in the first eight of 30 trials (Thut et al. 1996). Eight blocks were allocated to right-to-left, the other eight to leftto-right direction of transfer. With each type of transfer direction, four blocks involved proximal-to-proximal and the other four distal-to-distal transfer. The sequence of transfer direction and muscle group involved were partially counterbalanced within and between subjects. For each block, a different figure was presented. The figures consisted of the same three elements and were thus matched for linear extent and complexity (Fig. 1). Data acquisition lasted about $2 \mathrm{~h}$. After the first half of the experiment, subjects had a 10 -min break. Experimental blocks were separated by short rest periods when required. 


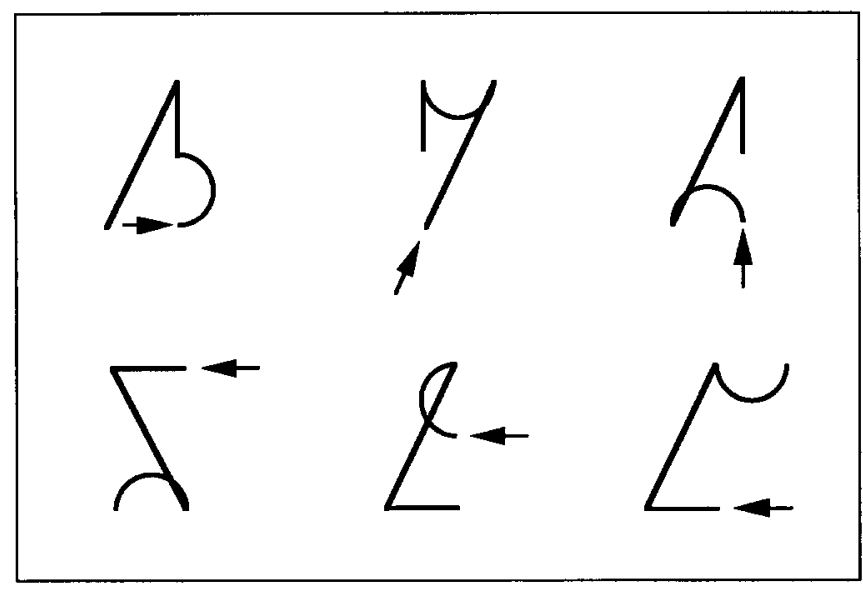

Fig. 1 Sample items of the 16 figures which had to be drawn. The arrows indicate the starting points and drawing directions

\section{Apparatus and data recording}

A detailed specification of the digitizing tablet used and the training trials to acquaint the subjects with it is provided elsewhere (Thut et al. 1996). Movement time, movement size and accuracy of extent were computed after each trial. Movement size was defined as the vertical movement extension, accuracy of extent as the absolute difference from the required size in the vertical dimension. Patients sometimes had difficulty maintaining the required minimal pressure on the pressure-sensing pen, leading to a trial with complete movements but incomplete data recording. These trials were rejected. In order not to confound early and late drawing performance, such trials were not replaced by later trials. Omitted trials accounted for $8.9 \%$ of all trials in patient O.G., for $13.3 \%$ in patient M.M. and for $13.7 \%$ in patient A.D.

\section{Data analysis}

Transfer performance was analysed for each patient individually and compared with the corresponding normal data (Thut et al. 1996) based on 26 right-handed, healthy males (age range 19-53 years, mean 33 years). Four transfer conditions were assessed: left-to-right distal, left-to-right proximal, right-to-left distal and right-to-left proximal. Compared with the patients, the healthy subjects performed fewer blocks (four instead of 16, one block per transfer condition), were presented with fewer figures (eight instead of 16) and performed more trials during TL ( 30 instead of eight). All other methodological aspects were identical.

\section{Results}

Averaged data for movement time, accuracy of extent and movement size are shown in Fig. 2 for all patients, trials and conditions. Conditions include performance without previous contralateral hand learning (original learning, OL) and after contralateral hand training (transfer learning, TL) at either non-dominant distal, non-dominant proximal, dominant distal or dominant proximal muscle groups.

The variable of interest was movement time during the first eight OL and TL trials. However, accuracy and

Fig. 2 Mean time until completion of each figure, mean accuracy of extent (absolute difference from the required size) and mean movement size (vertical dimension) are displayed as a function of the learning conditions [continuous lines trials without previous training $(\mathrm{OL})$, dashed lines trials after previous training in drawing the figure by the contralateral hand (TL)] and the muscle group involved (non-dominant distal, non-dominant proximal, dominant distal and dominant proximal) for all patients and the 30 trials

\section{MOVEMENT TIME [sec] \\ Non-dominant Dominant}

Distal Proximal Distal Proximal
ACCURACY OF EXTENT[mm]

\section{Non-dominant Dominant}

Distal Proximal Distal Proximal
MOVEMENT SIZE [mm]

\section{Non-dominant Dominant}

Distal Proximal Distal Proximal
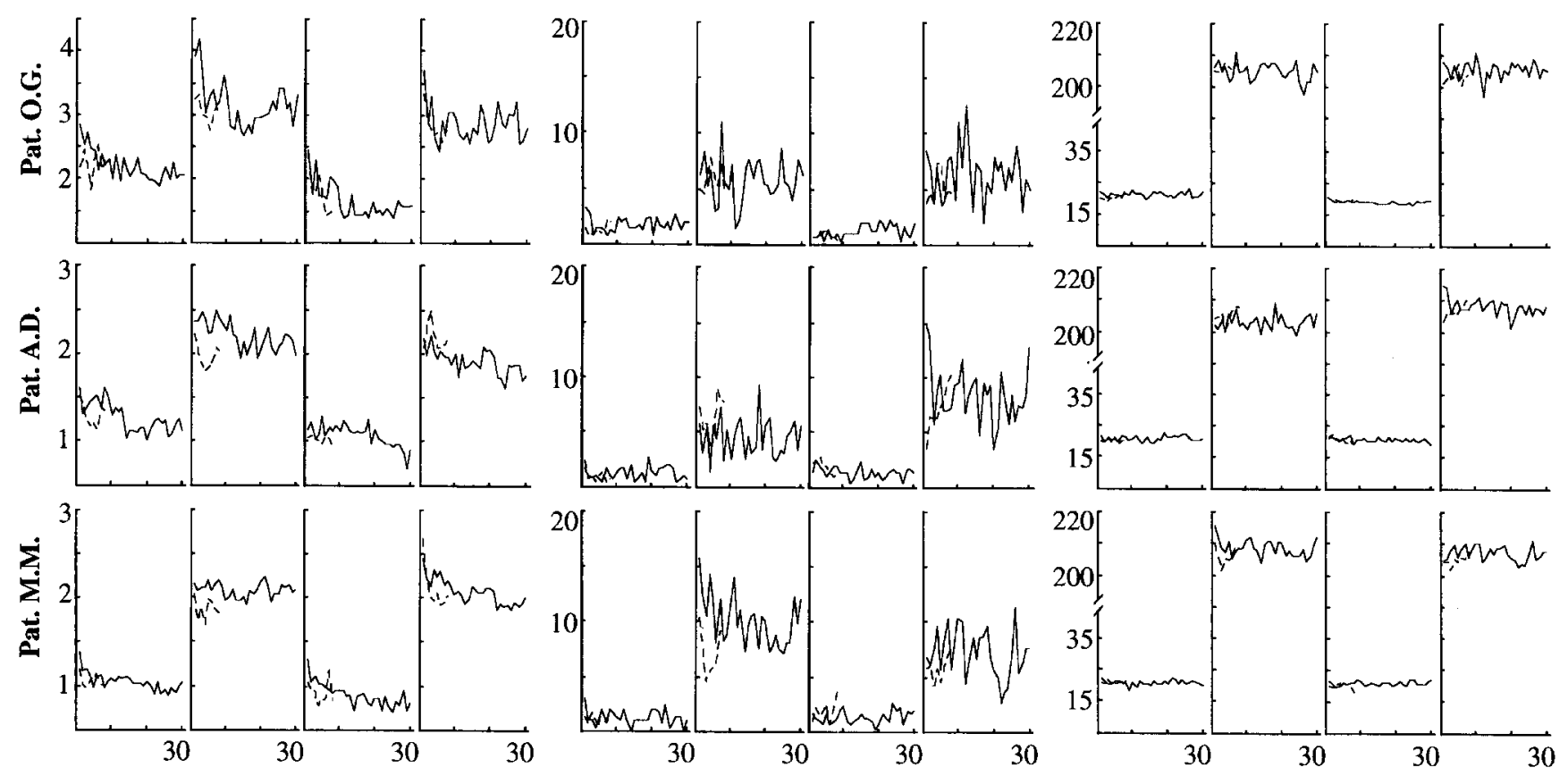

Number of Trials 
Table 1 Mean movement times (in ms), standard errors (SE) and variances (Var) over the first eight trials for each of the four effectors and for original learning $(O L)$ and transfer learning $(T L)$ in patients O.G., A.D. and M.M. Motor conditions with significant differences between OL and TL as assessed by $t$-tests are in bold type

\begin{tabular}{|c|c|c|c|c|c|c|c|c|c|}
\hline & & \multicolumn{4}{|c|}{ Non-dominant hand } & \multicolumn{4}{|c|}{ Dominant hand } \\
\hline & & \multicolumn{2}{|l|}{ Distal } & \multicolumn{2}{|l|}{ Proximal } & \multicolumn{2}{|l|}{ Distal } & \multicolumn{2}{|l|}{ Proximal } \\
\hline & & $\mathrm{OL}$ & TL & $\mathrm{OL}$ & TL & OL & TL & $\mathrm{OL}$ & $\mathrm{TL}$ \\
\hline \multirow[t]{3}{*}{ O.G. } & Mean & 2470 & 2231 & 3469 & $3059 *$ & 1967 & 1764 & 2963 & 2845 \\
\hline & $\mathrm{SE}$ & 82 & 112 & 97 & 93 & 99 & 93 & 123 & 111 \\
\hline & Var & $217 \mathrm{E} 3$ & $353 \mathrm{E} 3$ & $274 \mathrm{E} 3$ & $270 \mathrm{E} 3$ & 255E3 & $279 \mathrm{E} 3$ & $441 \mathrm{E} 3$ & $320 \mathrm{E} 3$ \\
\hline \multirow[t]{3}{*}{ A.D. } & Mean & 1459 & 1294 & 2369 & $1967 * * *$ & 1131 & 1020 & 2033 & 2207 \\
\hline & $\mathrm{SE}$ & 91 & 63 & 73 & 48 & 39 & 35 & 91 & 86 \\
\hline & Var & $214 \mathrm{E} 3$ & 94E3 & 139E3 & $74 \mathrm{E} 3$ & $41 E 3$ & $32 \mathrm{E} 3$ & $263 \mathrm{E} 3$ & 200E3 \\
\hline \multirow[t]{3}{*}{ M.M. } & Mean & 1139 & 1057 & 2135 & $1869 * *$ & 1054 & 934 & 2258 & 2101 \\
\hline & $\mathrm{SE}$ & 55 & 38 & 57 & 51 & 36 & 54 & 55 & 49 \\
\hline & Var & $81 \mathrm{E} 3$ & $35 \mathrm{E} 3$ & $104 E 3$ & $77 \mathrm{E3}$ & $31 \mathrm{E} 3$ & $78 \mathrm{E} 3$ & $79 \mathrm{E} 3$ & $78 \mathrm{E} 3$ \\
\hline
\end{tabular}

${ }^{*} P<0.05$; ${ }^{*} P<0.01$; ${ }^{* *} P<0.001$ ( $P$ values adjusted for multiple tests)

size had to be taken into account as a possible covariate of speed. Differences in movement time between TL and OL may be accounted for by changes in movement accuracy or movement size alone and may thus not be specific to transfer influences. To evaluate the interdependence of speed and the other two variables, Pearson correlations were performed for each patient and each of the four effectors separately. Raw data were used. None of the correlation coefficients was significant (ranging from $r=-0.14, P=0.3, n=58$ to $r=0.212, P=0.1, n=58)$, indicating that speed is not substantially influenced by accuracy or size in any condition. We therefore proceeded with analysis of movement time only.

\section{Individual transfer performance}

Table 1 shows mean movement times, standard errors and variances for early distal and proximal performance of either upper extremity (eight initial trials) in the OL and TL conditions. Screening of the data showed inhomogeneity of variance in patient A.D. Differences in variances exceeded the recommended factor of 4 (Howell 1992) for comparisons of dominant distal motor performance with other conditions. However, homogeneity of variance was obtained for all possible OL-TL comparisons. Intermanual transfer was therefore evaluated for each effector separately using unpaired $t$-tests with learning condition (OL, TL) as the grouping variable. Since a total of four tests were performed (OL-TL comparisons for each of the four effectors), the alpha error of $5 \%$ was adjusted to $1.3 \%$ (Bortz 1989). To make the analyses comparable between subjects, the same procedure was used for patients M.M. and O.G. All three patients (O.G.: $t=3.0, d f=58, \quad P<0.05$; A.D.: $t=4.7, d f=56$, $P<0.001$; M.M.: $t=3.5, d f=60, P<0.01$ ) performed movements significantly faster during TL compared with $\mathrm{OL}$ with the non-dominant proximal effector. No other comparison was significant.

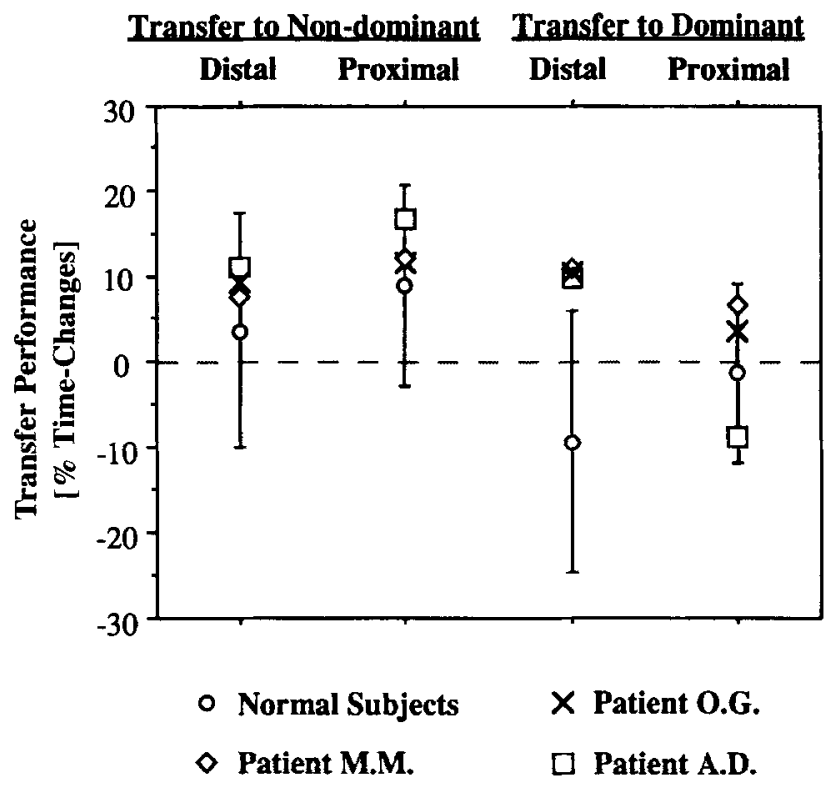

Fig. 3 Transfer performances for movement time based on relative performance changes during TL with $\mathrm{OL}$ at the identical effector as the reference for all transfer conditions and for all three patients and a group of 26 healthy subjects (Thut et al. 1996). Positive values correspond to a transfer advantage, that is, faster performance during TL than during OL. Error bars indicate the $99.9 \%$ confidence interval of the normal group. Only the first eight trials are included in the data

Individual comparisons with data from normals

Since patients proved to be slower than the healthy subjects for proximal (M.M., O.G., A.D.) and distal (O.G.) motor activity even during $\mathrm{OL}$, comparisons of transfer performance were performed on the basis of percentage changes in the TL condition relative to the corresponding OL condition. The formula was as follows: percentage change $=($ early $\mathrm{OL}$ minus early $\mathrm{TL})$ divided by early $\mathrm{OL}$ times 100 , where early OL or TL is the mean over the first eight trials assessed at the same effector. The pa- 
tients' transfer performance was judged to be affected when lying outside the $99.9 \%$ confidence interval of the normal group data.

The three patients' transfer performances based on the computed relative performance changes are depicted in Fig. 3. Confidence intervals of the corresponding normal group data are also presented. Positive values correspond to a transfer advantage, that is, faster performance during TL than during OL. As can be seen in Fig. 3, relative changes in movement time due to prior contralateral learning were within normal limits for transfer between proximal muscle groups as well as for transfer to the non-dominant hand for distal motor activity. Relative transfer changes of all three patients, however, exceeded the confidence interval of the healthy subjects at a $99.9 \%$ level for transfer between distal muscle groups from the non-dominant to the dominant side.

To rule out the possibility that differences in transfer performance in the dominant distal condition simply reflect age effects, the interrelation between age and transfer performance was examined in the control group. No significant correlation was found (Pearson correlation coefficient for dominant distal data $-0.008, n=26, P=0.97$ ).

\section{Discussion}

All three patients showed a proximal transfer benefit that was comparable to the transfer performance of the normal subjects, indicating that despite callosal agenesis or callosal damage the proximal transfer advantage was unaffected. This was interpreted to suggest that intermanual transfer between proximal muscle groups is facilitated by means of identical, extracallosal pathways in all three patients and the normals, most likely via ipsilateral motor pathways to proximal muscle groups. We conclude that compensatory mechanisms which might have become activated in the patients are rather unlikely to explain the results for the following reasons. It has been shown that compensation for impaired intermanual transfer is less effective when callosal damage occurs late in life after brain maturation has been completed (Lassonde et al. 1986) and that adjustment to interhemispheric disconnection has its limitations even in callosal agenesis subjects when speed of performance is analysed (Lassonde et al. 1988). Neither patient M.M., who suffered from callosal damage acquired late in life, nor the two agenesis patients would therefore be expected to reach completely normal transfer benefits when compensation is involved. One may argue that the weak evidence for left arm dominance of M.M. may raise difficulties in interpretation. Because our objective was to study whether there are transfer disturbances rather than differentiating between dominant and nondominant performance, we did not consider this a major problem.

This study makes a further point. Compared with the normal subjects, all three patients showed a superiority for non-dominant-to-dominant distal transfer. Since a disadvantage was observed for relearning at the domi- nant distal effector in the normals (Thut et al. 1996), the results were interpreted to reflect missing callosal inhibition. Because the normal group was on average younger than the three patients, differences in age might also have contributed to the result. If the superiority was due to age effects, however, transfer performance should also change with age in the control group, since all three patients were about as old as the oldest healthy subject. Because no relation was found, callosal effects are more likely to be involved. It has been argued that functional hemispheric specialization for language might partially evolve due to callosal mechanisms of an inhibitory nature (e.g. Moscovitch 1977; Cook 1986). Our results suggest that such a model might also be relevant to praxis at least when distal movements are performed. Motor learning may change functional asymmetries not only by the learning process itself but also by suppression of relearning in the contralateral hand through callosal inhibition. It is unclear, however, why there was more inhibition of the dominant motor system in the controls. From such a model, more pronounced inhibition of the nondominant system, which over time resulted in lateralization, would be expected. One may speculate that in the mature brain, the non-dominant motor system cannot be further suppressed since it is already at its lowest performance level, while the high performance level of the dominant system makes it vulnerable to influences of an inhibitory nature.

Several studies on intermanual transfer of training showed impaired transfer in patients with callosal damage, suggesting that callosal influences are facilitatory in nature (e.g. Lassonde et al. 1986; Levin et al. 1993). Furthermore, a series of studies using Poffenberger's visuo-motor reaction time paradigm (1912) revealed that interhemispheric transmission is asymmetric (e.g. Marzi et al. 1991) and that this might be due to asymmetric facilitation through callosal motor channels (Iacoboni and Zaidel 1995). While our conclusions are based on intermanual transfer of a distal multi-joint motor skill, transcallosal facilitation was inferred from studies on intermanual transfer of more complex tasks involving problem-solving strategies, i.e., form-board (e.g. Lassonde et al. 1986) and finger maze performance (e.g. Levin et al. 1993), or from research on transfer of distal single-joint motor commands (Iacoboni and Zaidel 1995). The present results thus suggest that callosal transfer mechanisms are task specific and differ even according to the level of motor processing (single-joint command versus multi-joint engram).

Acknowledgements This study was made possible by the Swiss National Science Foundation (grant no. 31-30123.90). We wish to thank Prof. Th. Gasser for statistical advice.

\section{References}

Bortz J (1989) Statistik für Sozialwissenschafter. Springer, Berlin Heidelberg New York, p 322

Brinkman J, Kuypers HGJM (1973) Cerebral control of contralateral and ipsilateral arm, hand and finger movements in the split-brain rhesus monkey. Brain 96: 653-674 
Bryden MP (1977) Measuring handedness with questionnaires. Neuropsychologia 15: 617-624

Colebatch JG, Gandevia SC (1989) The distribution of muscular weakness in upper motor neuron lesions affecting the arm. Brain 1 12: 749-763

Cook ND (1986) The brain code. Mechanisms of information transfer and the role of the corpus callosum. Methuen, New York

Gazzaniga MS, Bogen JE, Sperry RW (1967) Dyspraxia following division of the cerebral commissures. Arch Neurol 16: 606612

Gould HJ, Cusick CG, Pons TP, Kaas JH (1986) The relationship of corpus callosum connections to electrical stimulation maps of motor, supplementary motor, and the frontal eye fields in owl monkeys. J Comp Neurol 247: 297-325

Harrison DW (1991) Concurrent verbal interference of right and left proximal and distal upper extremity tapping. Acta Psychol 76: $121-132$

Howell DC (1992) Statistical methods for psychology. Duxbury Press, Belmont, Calif, p 308

Iacoboni M, Zaidel E (1995) Channels of the corpus callosum: evidence from simple reaction times to lateralized flashes in the normal and split brain. Brain 118: 779-788

Lassonde M, Sauerwein H, Geoffroy G, Décarie M (1986) Effects of early and late transection of the corpus callosum in children. Brain 109: 953-967

Lassonde M, Sauerwein H, McCabe N, Laurencelle L, Geoffroy G (1988) Extent and limits of cerebral adjustment to early section or congenital absence of the corpus callosum. Behav Brain Res 30: 165-181

Levin HS, Mattson AJ, Levander M, Lindquist CEH, Simard JM, Guinto FC, Lilly MA, Eisenberg HM (1993) Effects of transcallosal surgery in interhemispheric transfer of information. Surg Neurol 40: 65-74

Luria AR (1980) Higher cortical functions in man, 2nd edn. Basic Books, New York, p 421

Mack L, Rothi LJG, Heilman KM (1993) Hemispheric specialization for handwriting in right handers. Brain Cogn 21: 80-86
Marzi CA, Bisiacchi P, Nicoletti R (1991) Is interhemispheric transfer of visuomotor information asymmetric? Evidence from a meta-analysis. Neuropsychologia 29: 1163-1177

Mayer E, Hauert C-A, Pellizzer G, Stucchi N (1991) Coordinations bimanuelles dans un cas de dysconnexion calleuse. Rev Neuropsychol 1: 434-435

Meyer B-U, Röricht S, Gräfin von Einsiedel H, Kruggel F, Weindl A (1995) Inhibitory and excitatory interhemispheric transfers between motor cortical areas in normal humans and patients with abnormalities of the corpus callosum. Brain 118: 429440

Moscovitch M (1977) The development of lateralization of language functions and its relation to cognitive and linguistic development: a review and some theoretical speculations. In: Segalowitz SJ, Gruber FA (eds) Language development and neurological theory. Academic Press, New York, pp 193-211

Nakamura K, Naito H, Kurosaki T, Tamura Y (1971) Effect of polarizing currents on transcallosal postsynaptic potentials of cat pyramidal tract cells. Brain Res 35: 547-550

Pandya DN, Vignolo LA (1971) Intra- and interhemispheric projections of the precentral, premotor and arcuate areas in the rhesus monkey. Brain Res 26: 217-233

Parlow SE, Kinsbourne M (1989) Asymmetrical transfer of training between hands: implications for interhemispheric communication in normal brain. Brain Cogn 11: 98-113

Peters M, Murphy K (1992) Cluster analysis reveals at least three, and possibly five distinct handedness groups. Neuropsychologia $30: 373-380$

Poffenberger AT (1912) Reaction time to retinal stimulation with special reference to the time lost in conduction through nervous centres. Arch Psychol 23: 1-73

Steenhuis RE, Bryden MP (1989) Different dimensions of hand preference that relate to skilled and unskilled activities. Cortex 25: 289-304

Thut G, Cook ND, Regard M, Leenders KL, Halsband U, Landis $T$ (1996) Intermanual transfer of proximal and distal motor engrams in humans. Exp Brain Res 108: 321-327 